\title{
Truncation of the Projection Domain of MAP4 (Microtubule-Associated Protein 4) Leads to Attenuation of Microtubule Dynamic Instability
}

\author{
Sofy Permana ${ }^{1,2}$, Shin-ichi Hisanaga ${ }^{3}$, Yoko Nagatomo ${ }^{1}$, Junko Iida ${ }^{3}$, Hirokazu Hotani ${ }^{1}$, and \\ Tomohiko J. Itoh ${ }^{{ }^{*}}$ \\ ${ }^{1}$ Division of Biological Sciences, Graduate School of Science, Nagoya University, Furo-cho, Chikusa-ku, \\ Nagoya 464-8602, '2Department of Biology, Faculty of Mathematics and Natural Sciences, Brawijaya \\ University, JI. Veteran, Malang 65145, East-Java, Indonesia, and ${ }^{3}$ Department of Biological Sciences, \\ Graduate School of Science, Tokyo Metropolitan University, 1-1 Minami-Ohsawa, Hachiohji, Tokyo 192-0397, \\ Japan
}

\begin{abstract}
MAP4, a ubiquitous heat-stable MAP, is composed of an asymmetric structure common to the heatstable MAPs, consisting of an N-terminal projection (PJ) domain and a C-terminal microtubule (MT)-binding (MTB) domain. Although the MTB domain has been intensively studied, the role of the PJ domain, which protrudes from MT-wall and does not bind to MTs, remains unclear. We investigated the roles of the PJ domain on the dynamic instability of MTs by dark-field microscopy using various PJ domain deletion constructs of human MAP4 (PJ1, PJ2, Na-MTB and KDM-MTB). There was no obvious difference in the dynamic instability between the wtMAP4 and any fragments at $0.1 \mu \mathrm{M}$, the minimum concentration required to stabilize MTs. The individual MTs stochastically altered between polymerization and depolymerization phases with similar profiles of length change as had been observed in the presence of MAP2 or tau. We also examined the effects at the increased concentrations of $0.7 \mu \mathrm{M}$, and found that in some cases the dynamic instability was almost entirely attenuated. The length of both the polymerization and depolymerization phases decreased and "pause-phases" were occasionally observed, especially in the case of PJ1, PJ2 or Na-MTB. No obvious change was observed in the increased concentration of wtMAP4 and KDM-MTB. Additionally, the profiles of MT length change were quite different in $0.7 \mu \mathrm{M}$ PJ2. Relatively rapid and long depolymerization phases were sometimes observed among quite slow length changes. Perhaps, this unusual profile could be due to the uneven distribution of PJ2 along the MT lattice. These results indicate that the PJ domain of MAP4 participates in the regulation of the dynamic instability.
\end{abstract}

Key words: microtubule/dynamic instability/MAP4/projection domain/dark-field microscopy

\section{Introduction}

The microtubule (MT), which is a hollow polymer composed of $\alpha$ - and $\beta$-tubulin heterodimers, is a major member of eukaryotic cytoskeletons. It is widely accepted that this

\footnotetext{
*To whom correspondence should be addressed: Tomohiko J. Itoh, Division of Biological Sciences, Graduate School of Science, Nagoya University, Furo-cho, Chikusa-ku, Nagoya 464-8602, Japan.

Tel: +81-52-789-3542, Fax: +81-52-789-2989

E-mail: tjitoh@nucc.cc.nagoya-u.ac.jp

Abbreviations: AP sequence, assembly-promoting sequence; EGTA, [ethylenebis (oxyethylenenitrilo)] tetraacetic acid; GTP, guanosine-5'triphosphate; MAP, microtubule-associated protein; MAP2, microtubuleassociated protein 2; MAP4, microtubule-associated protein 4; MT, microtubule; MTB domain, microtubule-binding domain; PIPES, 1,4-piperazinediethanesulfonic acid; PJ domain, projection domain.
}

structure is involved in various essential cellular functions such as mitosis, organelle movements and determination of cellular morphology (Desai and Mitchison, 1997). For example, when the cell enters mitosis, the radial array of long and stable interphase MTs disassembles and is converted into the bipolar spindle composed of shorter and more labile MTs. This reorganization is thought to be due to a change in the "dynamic instability" of MTs (Cassimeris, 1999). Dynamic instability (Mitchison and Kirschner, 1984) is a characteristic behavior of individual MTs, both in vivo and in vitro, where alternating phases of relatively slow polymerization and rapid depolymerization occur independently in individual MTs (Horio and Hotani, 1986; Walker et al., 1988; Cassimeris et al., 1988; Sammak and Borisy, 1988; Schulze and Kirschner, 1988). The regulation of dynamic instability is important for the various MT activi- 
ties in living cells.

Various factors that regulate the dynamic instability of MTs have been identified so far (Desai and Mitchison, 1997; Mandelkow and Mandelkow, 1995; Kinoshita et al., 2002; Hashimoto, 2003). Several protein factors have been identified to be MT stabilizers (e.g., heat-stable MAP) or MT destabilizers (e.g., XKCM or Op18/stathmin) (Cassimeris, 1999; Anderson, 1999). Structural heat-stable MAP, which associates with the MTs surface, stimulates polymerization of tubulin and is supposed to be a major regulator of the dynamic instability (Olmsted, 1986; Hirokawa, 1994). It has been known that tau or MAP2 reduced catastrophe frequency and increased rescue frequency (Dreschel et al., 1992; Pryer et al., 1992; Kowalski and Williams, 1993). On the other hand, MAP4, a ubiquitous heat-stable MAP (Bulinski, 1994), strongly enhanced the rescue frequency without decreasing the catastrophe frequency (Ookata et al., 1995). Presumably, the mode of the binding of heat-stable MAP along MT lattice is important at least for the rescue, since it was demonstrated that MAP2 bound inhomogeneously onto MT surface, and that rescue occurred only at the sites where MAP2 density was high (Ichihara et al., 2001).

All the heat-stable structural MAPs including MAP2, tau and MAP4 have a common asymmetric molecular structure consisting of an amino-terminal projection (PJ) domain and a carboxyl-terminal microtubule-binding (MTB) domain (Lewis et al., 1988; Joly et al., 1989; Aizawa et al., 1989; West et al., 1991). Previous studies have concentrated on the MT-assembly properties of the MTB domain, which is almost identical among these heat-stable MAPs (Aizawa et al., 1991; Tokuraku et al., 1999), especially in the imperfect repeats of the AP (assembly-promoting) sequence of 18 amino acids. In contrast, the PJ domain, which protrudes from MT-wall and does not bind to MT, is completely different in length among the heat-stable MAPs. In addition, there is no homology in amino acid sequence among the PJ domains of the three MAPs, though it is more acidic than the MTB domain in all of them. Different properties among the heat-stable MAPs might be due to the difference in the PJ domain. The PJ domain of MAP4 is composed of three regions, the amino-terminal acidic region (Na-region), the multiple KDM-repeated sequence region (KDM-region) and the b-region, respectively (West et al., 1991). The $\mathrm{KDM}$-repeat region is a characteristic structure of the $\mathrm{PJ}$ domain of MAP4, and KDM-repeat is defined to be an imperfect 14 amino acid repeat $(\mathrm{KD}(\mathrm{M} / \mathrm{V}) \mathrm{X}(\mathrm{L} / \mathrm{P})(\mathrm{P} / \mathrm{L})$ XETEVALA) starting with $\mathrm{KD}(\mathrm{M} / \mathrm{V})$ as a highly conserved unit (Aizawa et al., 1990; Chapin et al., 1991; West et al., 1991). Although the primary structure of PJ domain has been well studied, the roles of the PJ domain on the regulation of MT dynamics or their organization are not well understood. MTs polymerized in the presence of MAP2, where the PJ domain was proteolytically removed, were much more resistant to a MT inhibitor, maytansine, than those polymerized in the presence of undigested MAP2 (Fellous et al., 1994). In tau, the N-terminal PJ domain was suggested to bind weakly to MTs (Gustke et al., 1994). Recently, PJ domain of MAP4 was shown to have an inhibitory activity to the MTB domain-induced bundling of MTs (Iida et al., 2002). These observations suggested that the PJ domain might also modify the activity of MTB domain in the polymerization of MTs. In addition, differential expression of the MAP2-variants with the different PJ domains during brain development suggests a distinct physiological role for the PJ domain of MAP2 (Kindler et al., 1990). Thus, a more detailed investigation is necessary for the role of PJ domain in MT dynamics.

In the present study, we analyzed the dynamic instability of MTs in the presence of various truncations of human MAP4 (1-1152 amino acid residues) with different lengths of the PJ domain. Here, we show that the truncation of the PJ domain from MAP4 leads to an attenuation of MT dynamics. This is direct evidence for the modification of the MT dynamics by the PJ domain of heat-stable MAPs.

\section{Materials and Methods}

\section{Chemicals and protein preparations}

Tubulin was purified from MT proteins of bovine or porcine brain by DEAE-Sepharose column chromatography using FPLC (Amersham Biosciences Co., USA) as described previously (Itoh et al., 1997). A wild type human MAP4 (1-1152 amino acid residues) (wtMAP4) or its truncation fragments, PJ1, PJ2, Na-MTB and KDM-MTB (Fig. 1) expressed in E. coli was purified as described previously (Iida et al., 2002) with minor modifications. Proteins were purified from the heat-stable fraction of bacterial cell lysates after boiling for five minutes in the presence of $0.5 \mathrm{M}$ $\mathrm{NaCl}$ and $0.1 \%(\mathrm{v} / \mathrm{v})$ of diisoprophyl fluorophosphate (DFP), followed by elution with $0.05-0.5 \mathrm{M}$ of $\mathrm{NaCl}$ using Mono-S column chromatography (Amersham Biosciences Co., USA). Salt was removed by dialyzing against PM buffer (100 mM PIPES, $\mathrm{pH}$ 6.9; $1 \mathrm{mM}$ EGTA and $0.5 \mathrm{mM} \mathrm{MgSO}{ }_{4}$ ). All protein preparations were frozen in liquid $\mathrm{N}_{2}$ and stored at $-80^{\circ} \mathrm{C}$ until use.

\section{Measurement of the total amount of MTs and bound MAPS}

The amount of MTs and bound MAPs at various conditions were estimated by ultracentrifugation followed by SDS-PAGE analysis. MTs were polymerized in PM buffer containing $1 \mathrm{mM}$ of GTP. After five minutes incubation at $37^{\circ} \mathrm{C}$ to allow the spontaneous polymerization of MTs from $30 \mu \mathrm{M}$ of tubulin, an equal volume of intact MAP4 or its mutants was added to make a final concentration of $15 \mu \mathrm{M}$ tubulin and $0.1-0.7 \mu \mathrm{M}$ of MAPs. The mixture was immediately spun at $40,000 \mathrm{rpm}$ for 30 minutes at $27^{\circ} \mathrm{C}$ using a TL-100 ultracentrifuge (Beckman Coulter Inc., USA) to precipitate MTs. After transferring the supernatants into another test tube, 


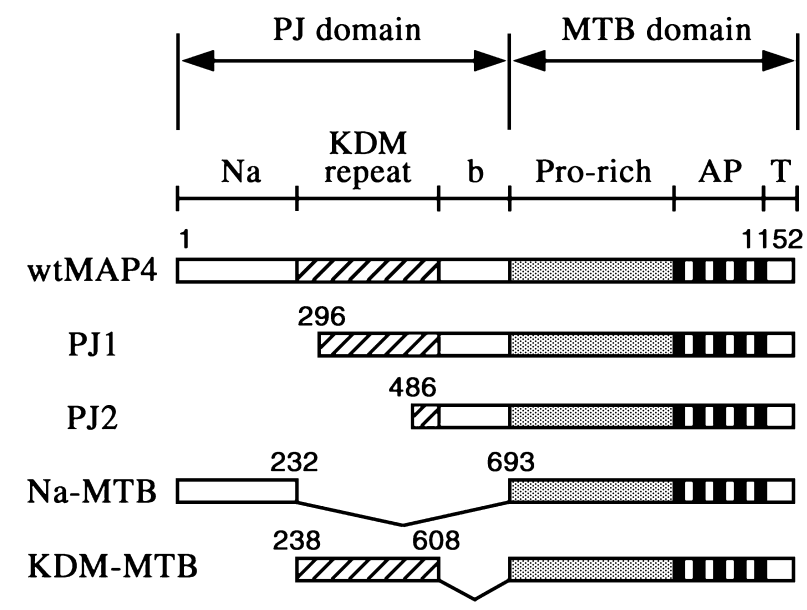

Fig. 1. Schematic structures of truncated MAP4 fragments used in this study. Full-length human MAP4 consists of 1152 amino acid residues as indicated. PJ1 lacks 9-295th residues, PJ2 lacks 9-485th residues, NaMTB lacks 233-692th residues and KDM-MTB lacks 9-237th and 609692th residues. Although PJ1, PJ2 and KDM-MTB possess the 1-8th amino acid residues, they are not shown in this diagram for the sake of clarity. Details in these constructs were described previously (Iida et al., 2002).

the pellet was resuspended in the same volume of PM buffer. The supernatants and pellet solutions were examined by SDS-PAGE $(10 \%(\mathrm{w} / \mathrm{v})$ polyacrylamide). After protein bands were stained with Coomassie brilliant blue R (CBB), gel images were incorporated into a Power Macintosh as PICT files using an image scanner (ScanJet 4c/T, Hewlett Packard, USA). Finally, the amounts of tubulin in polymers or the amount of bound MAPs were estimated by using program NIH-Image 1.61 .

\section{Observation of dynamic instability of MTs}

MT dynamics were observed with dark-field microscopy. MTs were polymerized in PM buffer containing $1 \mathrm{mM}$ GTP. After five minutes incubation at $37^{\circ} \mathrm{C}$ to allow the spontaneous polymerization of the MTs from $30 \mu \mathrm{M}$ of tubulin, an equal volume of wtMAP4 or its deletion mutants was added to make a final concentration of $15 \mu \mathrm{M}$ tubulin and $0.1-0.7 \mu \mathrm{M}$ of MAPs. Three minutes after mixing at room temperature $\left(27^{\circ} \mathrm{C}\right), 5 \mu \mathrm{l}$ of sample solution was transferred onto a glass slide. All the procedures for microscope observation and the analysis of dynamics were described previously (Itoh and Hotani, 1994), except that the microscope was put inside a temperature-controlled box (InterMedical, Japan) to keep the temperature at $27^{\circ} \mathrm{C}$.

\section{Miscellaneous}

Purity of the protein preparations was determined with SDS-PAGE according to Laemmli (1970). All protein concentrations were estimated by bichinchoninic acid (BCA) protein assay kit (Pierce Chemical Co., USA) using bovine serum albumin (BSA) as the standard. An unpaired t-test was used to make comparisons
Table I. EFFECTS OF PROJECTION DOMAIN DELETION OF MAP4 ON THE TOTAL AMOUNT OF POLYMERIZED MTS AND MT-BINDING

\begin{tabular}{lrccc}
\hline & $\mathrm{N}^{\mathrm{a}}$ & $\begin{array}{c}\text { Amount of MTs } \\
(\mu \mathrm{M})\end{array}$ & $\begin{array}{c}\text { Amount of bound } \\
\text { MAPs }(\mu \mathrm{M})\end{array}$ & $\begin{array}{c}\text { Ratio of bound } \\
\text { MAPs/tubulin } \\
(\mathrm{mol} / \mathrm{mol})\end{array}$ \\
\hline Control & 12 & $4.5 \pm 0.7$ & - & - \\
wtMAP4 & 6 & $5.9 \pm 1.9^{\mathrm{b}}$ & $0.25 \pm 0.11$ & $0.05 \pm 0.02$ \\
PJ1 & 5 & $5.5 \pm 1.3^{\mathrm{b}}$ & $0.32 \pm 0.15$ & $0.06 \pm 0.03$ \\
PJ2 & 9 & $6.4 \pm 1.1^{\mathrm{b}}$ & $0.60 \pm 0.19^{\mathrm{c}}$ & $0.09 \pm 0.03^{\mathrm{d}}$ \\
Na-MTB & 3 & $5.4 \pm 0.5^{\mathrm{b}}$ & $0.33 \pm 0.10$ & $0.06 \pm 0.03$ \\
KDM-MTB & 3 & $6.0 \pm 1.6^{\mathrm{b}}$ & $0.35 \pm 0.13$ & $0.06 \pm 0.02$ \\
\hline
\end{tabular}

The amounts of MTs polymerized in $15 \mu \mathrm{M}$ tubulin in the presence of $0.7 \mu \mathrm{M}$ of various MAP4 mutants were estimated by ultracentrifugation followed by SDS-PAGE analysis. The amount of bound MAPs and molar ratios of bound MAP4 mutants were also obtained. These results indicate that wtMAP4 and projection domain-deletion mutants enhance the polymerization of MTs. Control MTs were polymerized in the absence of MAP4 constructs.

${ }^{\text {a }} \mathrm{N}$ is the number of experiments.

${ }^{\mathrm{b}}$ Amount of MTs significantly increased in the presence of $0.7 \mu \mathrm{M}$ MAPs than that polymerized without MAPs (control) $(p>0.01)$.

${ }^{c}$ Amount of bound MAPs is significantly higher than wtMAP4 $(p>0.01)$.

d Ratio of bound MAPs/tubulin is significantly higher than wtMAP4/tubulin $(p>0.01)$.

between the wtMAP4 values and each mean with Macintosh software, Kaleida Graph (Synergy Software, USA).

\section{Results}

\section{Polymerization of MTs in the presence of wtMAP4 or its PJ domain-deletion fragments}

Several PJ domain deletion mutants induce MT bundling (Iida et al., 2002). Because MT bundles disturb the observation of the length changes of single MTs by dark-field microscopy, we must observe MT dynamics under the appropriate condition where MTs do not form bundles. We first scanned the concentration of each truncation mutant (Fig. 1) to determine the upper limit of concentration for the observation. The enhancement of MT polymerization and bundling induced by wtMAP4 or its fragments was observed by dark-field microscopy. Slight increases in the number of MTs per field were observed by adding these MAPs at the concentration of $0.1 \mu \mathrm{M}$ or more. The number of MTs apparently increased with the increase in the concentration of MAPs up to $2.4 \mu \mathrm{M}$ (data not shown), though bundles of MTs were occasionally observed in the presence of several fragments, especially in the case of PJ2, at concentrations higher than $0.7 \mu \mathrm{M}$. Thus, we did not analyze the dynamics of individual MTs in the presence of any MAP4 constructs at concentrations higher than $0.7 \mu \mathrm{M}$. In addition, we could not examine the construct, MTB, which lacked the entire length of PJ domain, since it formed huge MT-bundles even at lower concentrations (Iida et al., 2002). 
Then we estimated the amount of MTs by a sedimentation assay. As shown in Table I, the total amount of sedimented MTs slightly increased by the addition of $0.7 \mu \mathrm{M}$ of wtMAP4 or its mutants. A remarkable increase of polymerized MT was detected only in the presence of PJ2. The concentrations of bound MAPs were also obtained by measuring MAPs co-sedimented with MTs. Under the present condition, concentrations of bound MAPs ranged between $0.25 \mu \mathrm{M}$ and $0.35 \mu \mathrm{M}$ among the constructs including wtMAP4, PJ1, Na-MTB and KDM-MTB, where no significant difference was detected between them. On the other hand, the concentration of bound PJ2 was $0.60 \pm 0.19 \mu \mathrm{M}$, which was significantly higher than that of the other MAPs (Table I). This higher concentration of PJ2 recovered in precipitate was not simply due to the increased amount of MTs, because the PJ2/tubulin ratio was also significantly higher than for wtMAP4 (Table I). These results indicated that the partial deletion of $\mathrm{N}$-terminal of the PJ domain did not affect the binding ability to MTs or enhance MT polymerization, but that the longer deletions of the PJ domain increased MT binding activity such as observed here for PJ2 or MTB (Kawachi et al., 2003).

\section{Observation of MT dynamics by dark-field microscopy}

To determine the role of the PJ domain of MAP4 on MT dynamics, we observed the dynamic instability of MTs in the presence of various concentrations of wtMAP4 or its fragments. As described previously (Itoh and Hotani, 1994), a final concentration of $15 \mu \mathrm{M}$ of tubulin was appropriate to observe the individual MTs whose ends were easily distinguished. Both ends of the MT could be distinguished from the difference in their growth rates. Since the MT dynamics mainly changes at the plus end when MAPs are added (Horio and Hotani, 1986; Itoh and Hotani, 1994; Ookata et al., 1995; Trinczek et al., 1995), we intently examined the dynamic instability profiles of the plus end for 15 minutes.

In the absence of any MAP4 recombinants, approximately half of the MTs disappeared without undergoing any rescue within a few minutes after setting specimens on the microscope stage, but the other $50 \%$ of the MTs underwent several rescues and could be followed at least 15 minutes. This property of MTs was slightly different from that reported previously in the absence of MAPs (Itoh and Hotani, 1994; Ookata et al., 1995). This difference is the result of improvements in the experimental conditions. We kept the temperature of the whole microscope at $27^{\circ} \mathrm{C}$. The temperature-dependent increase in the rescue frequency was consistent with the earlier results by Walker et al. (1989).

\section{MT dynamics in the presence of wtMAP4}

In the presence of $0.1 \mu \mathrm{M}$ of wtMAP4, which is the minimum concentration required to stabilize MTs, almost all the depolymerization phases were accompanied by the rescue, so that the majority MTs could be observed for at least 15 minutes. Fig. 2a shows typical profiles of the change in MT length at the plus end in the presence of $0.1 \mu \mathrm{M}$ wtMAP4. The duration of the polymerization phases ranged between two to seven minutes and catastrophe occurred around two to five times within the 15 minutes of observation in these MTs. These profiles are almost identical to those in the presence of small amounts of crude MAPs (Itoh and Hotani, 1994) or native MAP2 (Itoh et al., 1997). As shown in Fig. 4 , the polymerization velocity increased significantly compared to the no MAPs condition. The depolymerization velocity also increased slightly but the difference was not significant. The polymerization distance increased 1.4-fold, and the depolymerization distance decreased to $67 \%$ (Fig. 5 ). These results indicate that wtMAP4 expressed in $E$. coli stabilizes MTs by suppressing catastrophe and enhancing rescue. The MT-stabilizing activity of this MAP4 is slightly different from that observed in "intact MAP4" isolated from HeLa cells in interphase, which did not decrease catastrophe frequency (Ookata et al., 1995).

The increase of the concentration from $0.1 \mu \mathrm{M}$ to $0.7 \mu \mathrm{M}$ did not change the general profile of the dynamic instability except for the slight decrease in the polymerization velocity and increase in the depolymerization velocity (Fig. 3a, Fig. 4). The decrease in polymerization velocity would be due to the decrease in free tubulin concentration by increasing the amount of polymerized tubulin (Itoh and Hotani, 1994). Both the polymerization and depolymerization distances did not change even upon the increase in the concentration of wtMAP4 (Fig. 5).

\section{All MAP4 fragments similarly stabilized MTs as wtMAP4 at low concentration}

We examined the dynamic instability in the presence of various MAP4 fragments, PJ1, PJ2, Na-MTB or KDM-MTB, at $0.1 \mu \mathrm{M}$, and obtained profiles of the dynamic instability similar to those in the presence of wtMAP4 (Fig. 2b-e). All the MTs predominantly alternated between the polymerization phases and depolymerization phases in the presence of these MAP4 mutants. When comparing the parameters obtained in the presence of $0.1 \mu \mathrm{M}$ of various MAP4 fragments with those obtained in wtMAP4, no significant difference was detected in either the polymerization or the depolymerization velocities (gray columns in Fig. 4). The polymerization distance and the depolymerization distance also did not change significantly by any deletions of the PJ domain (gray columns in Fig. 5). These results indicate that all the PJ domain-deletion mutants similarly stabilize MTs at $0.1 \mu \mathrm{M}$, the minimum concentration required to stabilize MTs, as wtMAP4 regardless of the truncated domains. 

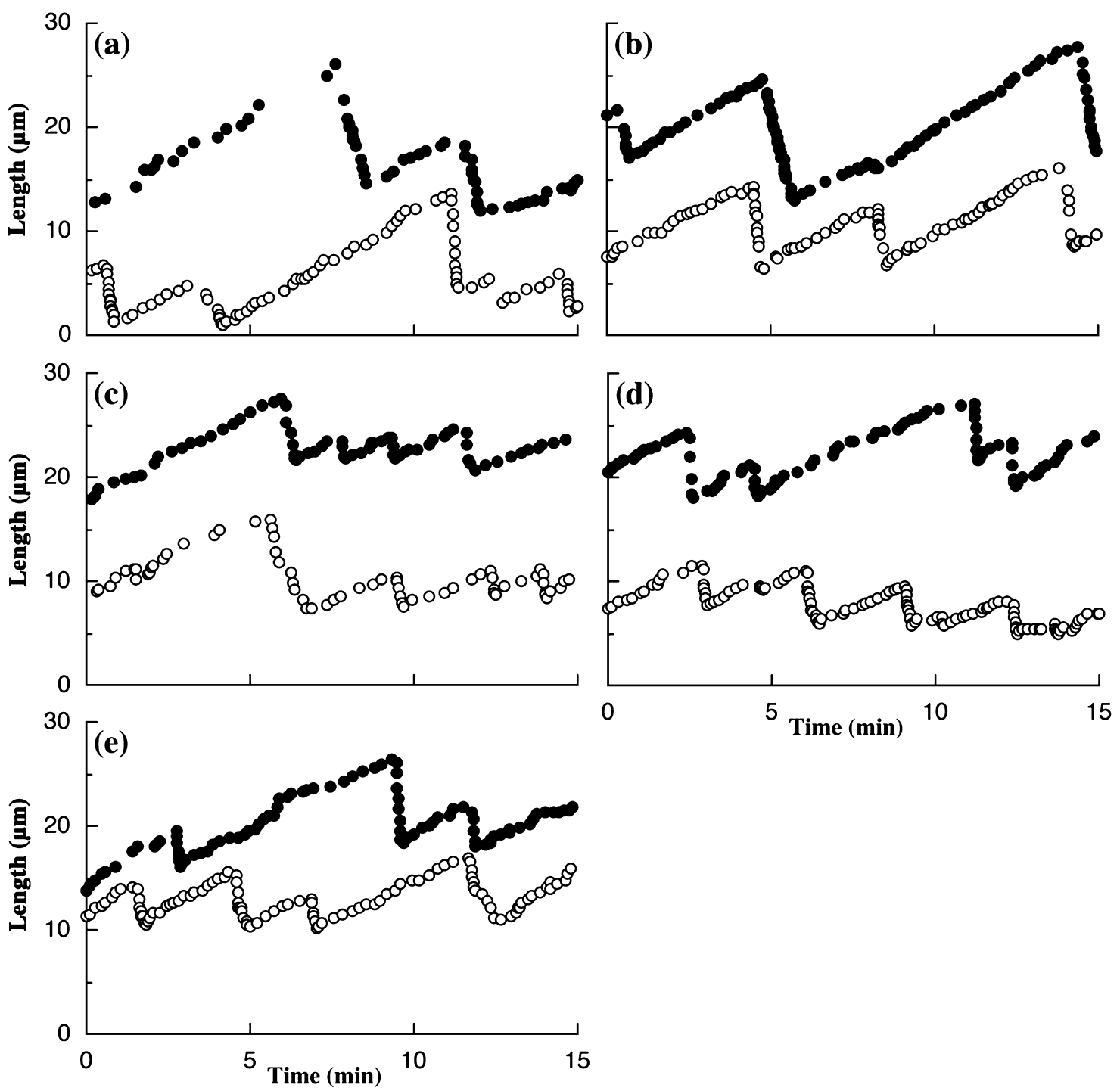

Fig. 2. Dynamic instability profiles of MTs at plus end in the presence of $0.1 \mu \mathrm{M}$ MAP4 fragments. Profiles of two representative MTs are shown with open and closed circles: (a) wtMAP4, (b) PJ1, (c) PJ2, (d) Na-MTB, (e) KDM-MTB. Tubulin concentration is $15 \mu$ M. Each data point was obtained by determining the distance between MT end and an arbitrary fixed point on the MT. From both ends of a MT, the more dynamic end with the higher velocity of polymerization and higher frequencies of catastrophe and rescue was defined to be the plus end (Itoh and Hotani, 1994). There is no obvious difference in the profiles of dynamic instability among the PJ domain truncations of MAP4.

\section{PJ domain truncation affected MT dynamics at increased concentration}

When we observed the dynamic instability of MTs with various MAP4 fragments at $0.7 \mu \mathrm{M}$, which was the upper limit of the concentration of MAP4 fragments where MTs did not form bundles by any mutants, obvious differences were observed between wtMAP4 and several MAP4 fragments. As shown in Fig. $3 \mathrm{~b}$ to $\mathrm{d}$, the profiles in the presence of $0.7 \mu \mathrm{M}$ of PJ1, PJ2 or Na-MTB were quite different from wtMAP4. The length of individual polymerization and depolymerization phases obviously decreased, as was observed with the high concentrations of brain crude MAPs preparation (Itoh and Hotani, 1994). Different from the case of brain crude MAPs, a "pause phase", where no significant change in length $(<0.3 \mu \mathrm{m}$ under our optical microscope resolution) was observed, was occasionally detected as indicated by asterisks in Fig. 3b-d. The duration of such "pause phases" ranged between 20 to $25 \%$ in $0.7 \mu \mathrm{M}$ PJ1, PJ2 or Na-MTB (Table II). In addition, the depolymerization velocity appeared to decrease in these three fragments (filled columns in Fig. 4). At the increased concentration of Na-MTB, both the polymerization distance and the depolymerization distance decreased to $62 \%$ of those for wtMAP4. 

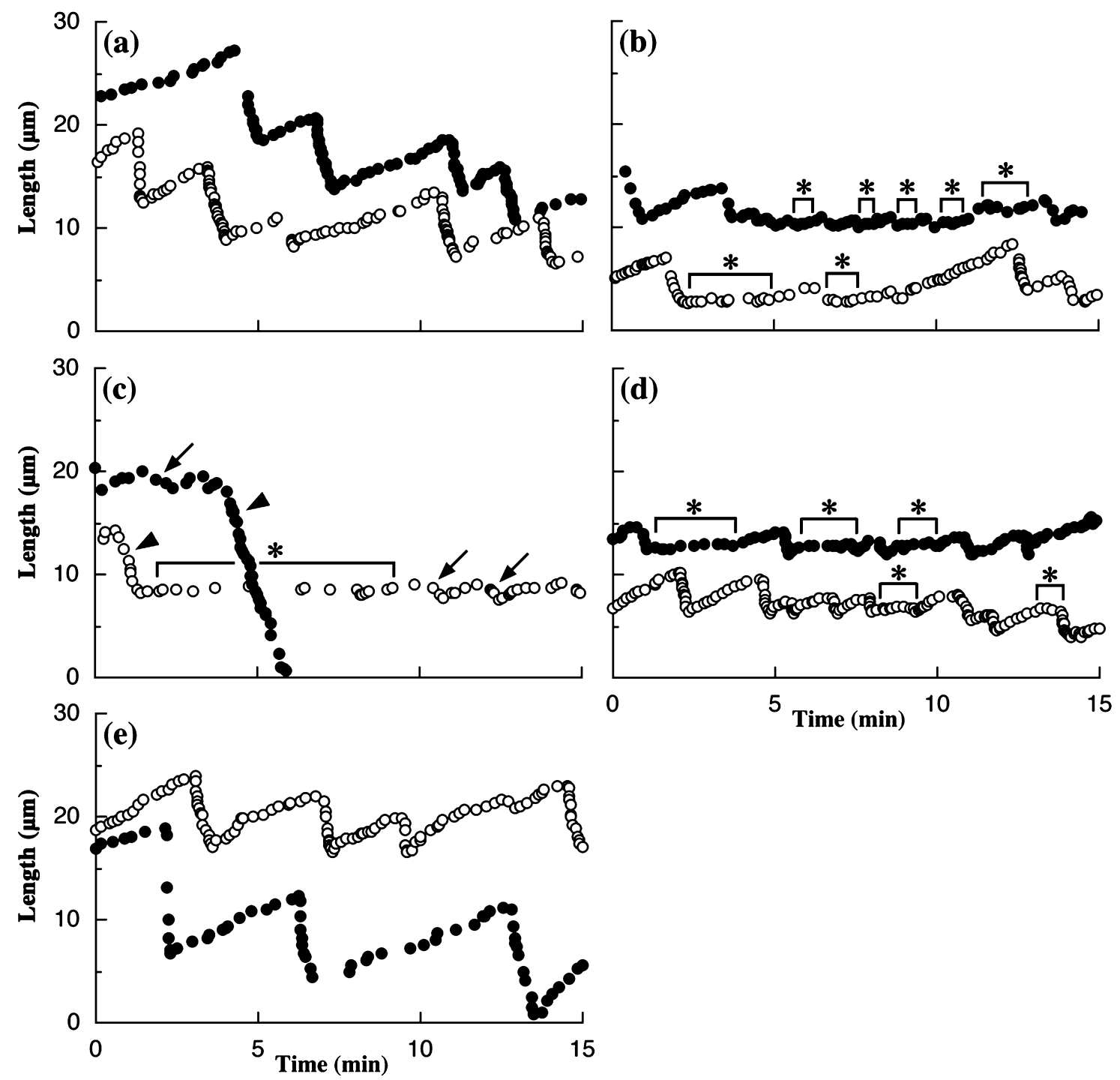

Fig. 3. Dynamic instability profiles of MTs at plus end in the presence of $0.7 \mu \mathrm{M}$ MAPs. Profiles of two representatives MTs are shown with open and closed circles: (a) wtMAP4, (b) PJ1, (c) PJ2, (d) Na-MTB, (e) KDM-MTB. Tubulin concentration is $15 \mu \mathrm{M}$. Each data point was obtained by determining the distance between MT end and an arbitrary fixed point on the MT. From both ends of a MT, the more dynamic end with the higher velocity of polymerization and higher frequencies of catastrophe and rescue was defined to be the plus end (Itoh and Hotani, 1994). Compared to the profiles of wtMAP4, obvious attenuation of dynamic instability was obtained in the presence of $0.7 \mu \mathrm{M}$ PJ1, PJ2 or Na-MTB, and "pause-phases" (indicated by asterisks) was occasionally observed. Especially, the "long" depolymerization phases (indicated by arrowheads) were rarely observed among the frequent slow "short" depolymerization phases (indicated by arrows) only in the presence of PJ2.

More obvious changes for these two parameters were obtained in the presence of $0.7 \mu \mathrm{M}$ of PJ1 or PJ2 (filled columns in Fig. 5). Consequently, the dynamic instability was attenuated by decrease in both polymerization and depolymerization velocities, and increase in both catastrophe and rescue frequencies at increased concentration; individual MTs became more static with these three MAP4 fragments than in wtMAP4. These observations indicate that partial removal of PJ domain from MAP4 affects the stability of MTs. We can conclude that the PJ domain of MAP4 parti- cipates in the dynamic instability of MTs, and that the effects of deletion appeared only when MAPs concentration is increased. However, these effects would be domaindependent as evidenced by the result that no obvious differences were detected between wtMAP4 and KDM-MTB at $0.7 \mu \mathrm{M}$ either in the dynamic profiles (Fig. 3e) or in the parameters of dynamic instability (Fig. 4 and Fig. 5). This suggests that the KDM-region is sufficient for the regulatory effects of the entire PJ domain on MT dynamics. Because dynamic instability was attenuated in the presence 

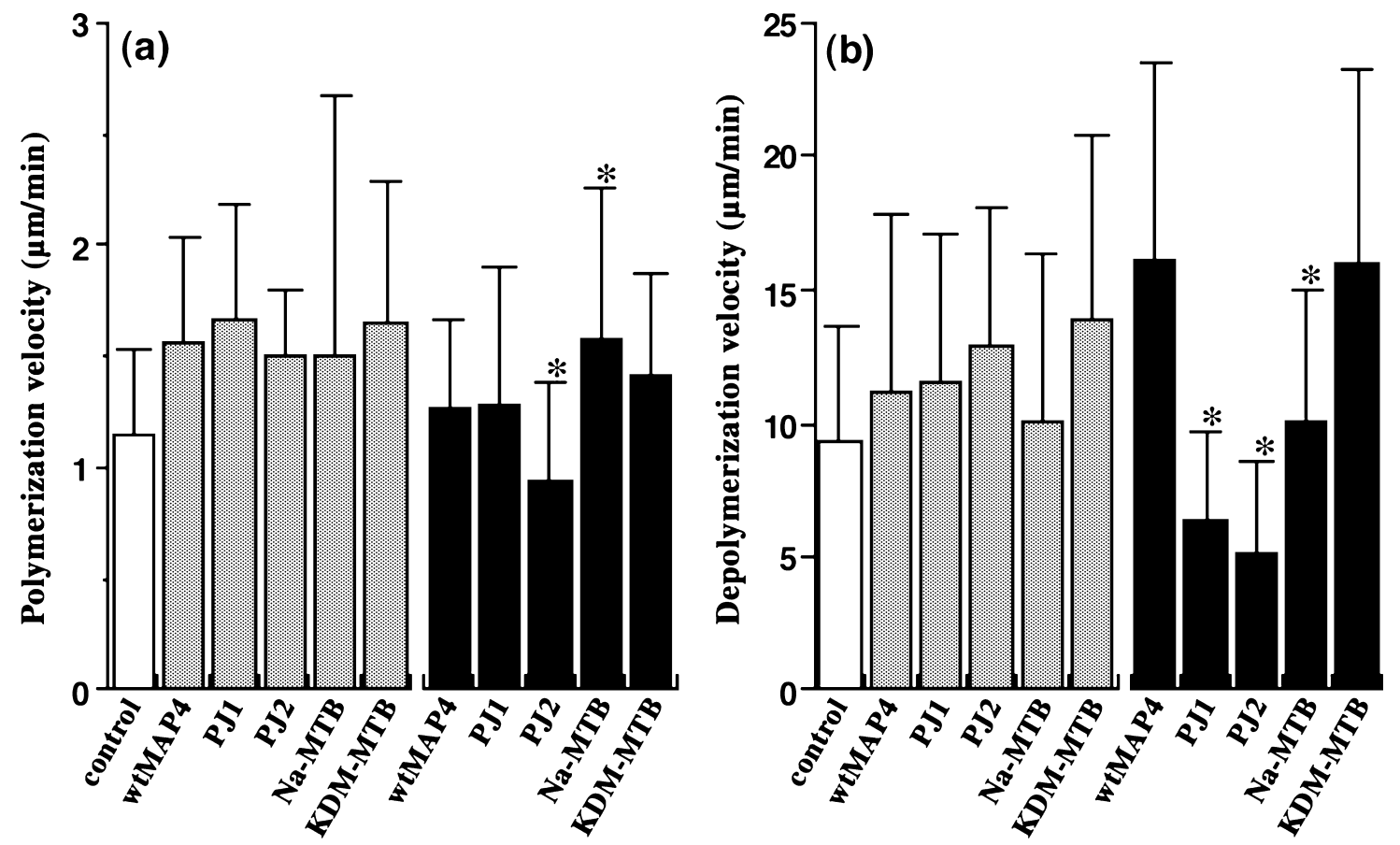

Fig. 4. Polymerization and depolymerization velocity of individual MTs in the presence of various PJ domain truncation mutants of MAP4. MT polymerization velocity $(a)$ and MT depolymerization velocity $(b)$ were obtained from direct observation of MTs using dark-field microscopy. $0.1 \mu$ M: gray columns; $0.7 \mu \mathrm{M}$ : filled columns. Numbers of phases used in these calculations are summarized in Table II. Data shown are means \pm S.D. Statistical significance, $* p<0.01$.
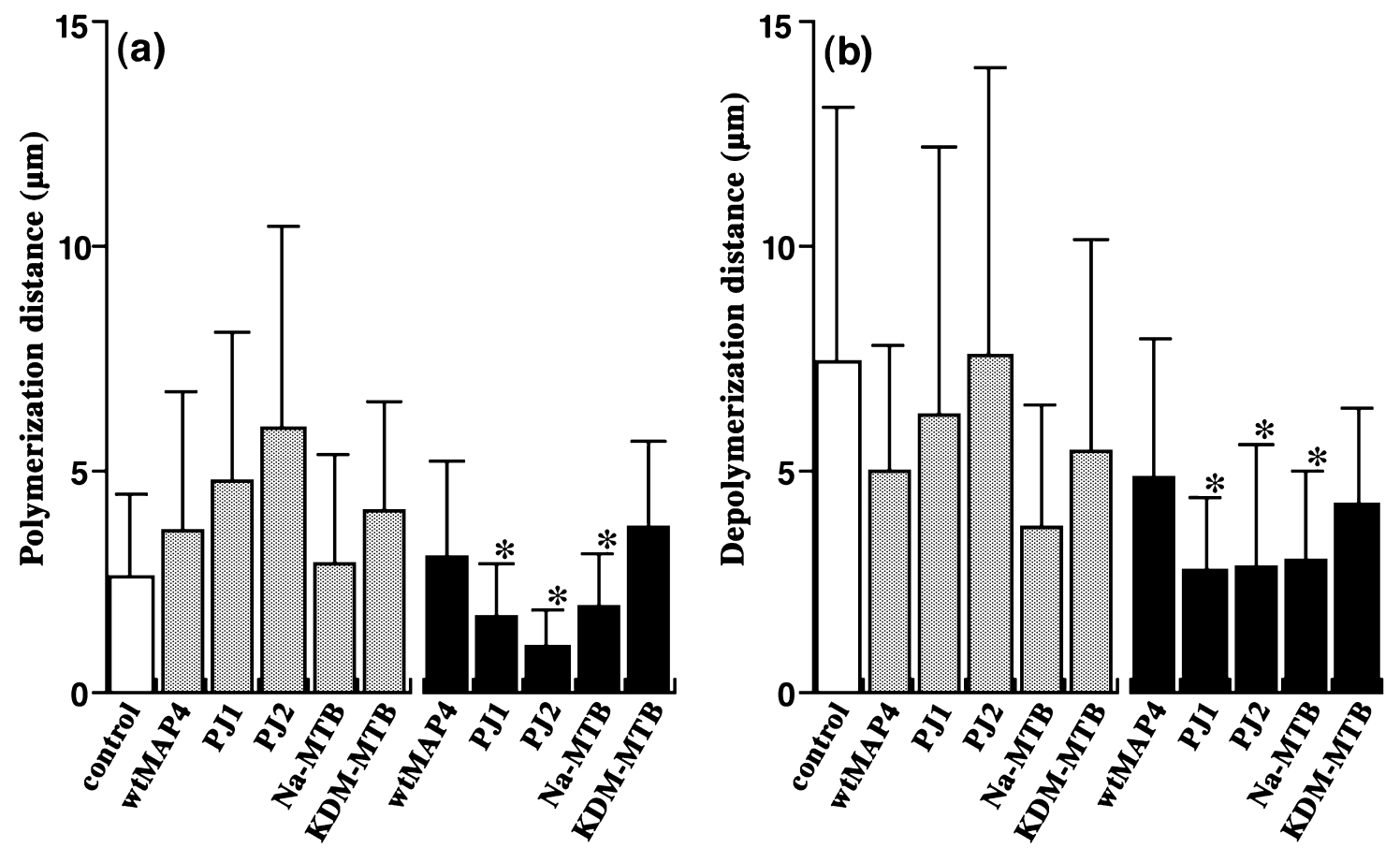

Fig. 5. Polymerization and depolymerization distance of individual MTs in the presence of various PJ domain truncation mutants of MAP4. MT polymerization distance $(a)$ and MT depolymerization distance $(b)$ were obtained from the direct observation of MTs using dark-field microscopy. $0.1 \mu \mathrm{M}$ : gray columns; $0.7 \mu \mathrm{M}$ : filled columns. Numbers of phases used in these calculations are summarized in Table II. Data shown are means \pm S.D. Statistical significance; $* p<0.01$. 
Table II. OBSERVATION OF MT DYNAMICS IN THE PRESENCE OF VARIOUS PJ DOMAIN-DELETION MUTANTS OF MAP4

\begin{tabular}{|c|c|c|c|c|c|c|c|c|}
\hline & $\begin{array}{c}\text { Concentration } \\
(\mu \mathrm{M})\end{array}$ & $\mathrm{N}_{\mathrm{MT}}$ & $\begin{array}{c}\text { Total time } \\
\text { (min) }\end{array}$ & $\mathrm{N}_{\mathrm{p}}$ & $\mathrm{N}_{\text {cat }}$ & $\mathrm{N}_{\mathrm{d}}$ & $\mathrm{N}_{\text {res }}$ & $\begin{array}{c}\text { Fraction of } \\
\text { pause }(\%)\end{array}$ \\
\hline Control & 0 & 18 & 139 & 36 & 33 & 36 & 23 & 0 \\
\hline \multirow{2}{*}{ wtMAP4 } & 0.1 & 8 & 120 & 42 & 34 & 34 & 33 & 0 \\
\hline & 0.7 & 9 & 129 & 43 & 38 & 39 & 35 & 0 \\
\hline \multirow{2}{*}{ PJ1 } & 0.1 & 8 & 120 & 37 & 29 & 30 & 29 & 0 \\
\hline & 0.7 & 9 & 120 & 45 & 32 & 37 & 36 & 25.2 \\
\hline \multirow{2}{*}{ PJ2 } & 0.1 & 8 & 120 & 25 & 18 & 19 & 17 & 0 \\
\hline & 0.7 & 15 & 131 & 50 & 44 & 54 & 40 & 23.2 \\
\hline \multirow{2}{*}{ Na-MTB } & 0.1 & 10 & 129 & 50 & 46 & 46 & 40 & 0 \\
\hline & 0.7 & 10 & 131 & 62 & 48 & 52 & 51 & 19.6 \\
\hline \multirow{2}{*}{ KDM-MTB } & 0.1 & 10 & 128 & 43 & 37 & 37 & 36 & 0 \\
\hline & 0.7 & 8 & 120 & 38 & 32 & 34 & 32 & 0 \\
\hline
\end{tabular}

$\mathrm{N}_{\mathrm{MT}}$ is the number of MTs employed for the statistics. $\mathrm{N}_{\mathrm{p}}$ is the total number of polymerization phase. $\mathrm{N}_{d}$ is the total number of depolymerization phase. $\mathrm{N}_{\text {cat }}$ is the total number of catastrophes, and $\mathrm{N}_{\text {res }}$ is the total number of rescues. Fraction of pause is obtained by dividing the sum of pause time by total observation time. Pause phase was observed only in $0.7 \mu \mathrm{M}$ of PJ1, PJ2 or Na-MTB.

Note that $\mathrm{N}_{\text {res }}$ is remarkably smaller than $\mathrm{N}_{\mathrm{d}}$ only in the control and in $0.7 \mu \mathrm{M}$ PJ2. This indicates that a significant number of depolymerization phases was not followed by rescue in these conditions.

of PJ1, which consists of almost all KDM-region and the bregion, it is possible that b-region negatively regulates the effect of the KDM-region.

\section{Two distinct depolymerization phases were observed in the presence of $0.7 \mu \mathrm{M} \mathrm{PJ} 2$}

The profiles of the dynamic instability in the presence of $0.7 \mu \mathrm{M}$ of PJ2 (Fig. 3c) can be classified into a different category from those in the increased concentration of PJ1 or Na-MTB (Fig. 3b, d). Almost all length changes were quite short in both the polymerization phase or depolymerization phase as indicated by arrows in Fig. 3c, but, different from PJ1 or Na-MTB, some depolymerization phases occur over long distances in the presence of PJ2 (indicated by arrowheads in Fig. 3c) even though the same MT showed a static profile at other times. The average polymerization distance decreased to $34 \%$ and the average depolymerization distance decreased to $60 \%$ in $0.7 \mu \mathrm{M}$ PJ2 (Fig. 5). Although the lengths of depolymerization phases were obviously shorter in most cases with an increased PJ2 concentration than those in the increased PJ1 or Na-MTB concentration (compare Fig. $3 \mathrm{c}$ with $3 \mathrm{~b}$ or $3 \mathrm{~d}$ ), the average depolymerization distance was higher (Fig. 5) than that expected from the apparently flat profile of the entire length change profile. This discrepancy was due to the presence of infrequent long depolymerization phases.

To determine the property of MT dynamics in the presence of $0.7 \mu \mathrm{M}$ of $\mathrm{PJ} 2$, the infrequent long depolymerization phases must be considered more precisely. When a MT underwent such long depolymerization, it occasionally disappeared without rescue (see Table II), so that the average half-life of MT tended to be shorter than those in the other
Table III. COMPARISON OF THE VELOCITIES BETWEen "short" and "long" DEPOLYMERIZATION PHASES IN wTMAP4 AND IN PJ2

\begin{tabular}{lll}
\hline & $\begin{array}{c}\text { Velocity of "short" phase } \\
(\mu \mathrm{m} / \mathrm{min})\end{array}$ & $\begin{array}{c}\text { Velocity of "long" phase } \\
(\mu \mathrm{m} / \mathrm{min})\end{array}$ \\
\hline \multirow{2}{*}{ wtMAP4 } & $17.1 \pm 8.9(\mathrm{~N}=15)$ & $15.4 \pm 6.4(\mathrm{~N}=24)$ \\
& $(2.0 \pm 0.6 \mu \mathrm{m})^{\mathrm{a}}$ & $(6.6 \pm 2.8 \mu \mathrm{m})^{\mathrm{a}}$ \\
PJ2 & $4.4 \pm 3.2^{\mathrm{b}}(\mathrm{N}=42)$ & $7.4 \pm 3.6(\mathrm{~N}=12)$ \\
& $(1.6 \pm 0.7 \mu \mathrm{m})^{\mathrm{a}}$ & $(6.7 \pm 4.0 \mu \mathrm{m})^{\mathrm{a}}$ \\
\hline
\end{tabular}

The lengths of depolymerization phases were categorized into "short" (shorter than or equal to $3 \mu \mathrm{m}$ ) and "long" (longer than $3 \mu \mathrm{m}$ ). ${ }^{a}$ The average lengths of the "short" and "long" phases are shown in parentheses. bThe velocity of depolymerization for "short" phases in the presence of 0.7 $\mu \mathrm{M}$ PJ2 was significantly slower than that for "long" phases (indicated by asterisks; $p>0.01$ ), while no significant difference was detected between them in wtMAP4.

MAP4 fragments. In addition, the velocity during such large excursions seemed to be faster than during the short depolymerization phases (arrows in Fig. 3c) in the apparently static phases. To distinguish the two types of depolymerization phases in more detail, we independently calculated the depolymerization velocity of short phases in the apparently static phases and that of long phases. The average velocity obtained from shorter depolymerization phases was significantly slower than that from those with longer excursion, while depolymerization velocity in wtMAP4 did not change regardless of the phase length (Table III). In these cases, we set the boundary between short and long phases at $3 \mu \mathrm{m}$ for analyzing the data both of wtMAP4 and PJ2, simply because the lengths of the shorter depolymerization phases observed in the presence of $0.7 \mu \mathrm{M}$ of PJ2 were always less than $3 \mu \mathrm{m}$. These results suggested that two distinct regions exist in a MT, such that depolymerization easily stops in the 
slowly depolymerizing region, but is very difficult to stop in the rapidly depolymerizing region. Co-presence of the long depolymerization phase with the static dynamic instability within a MT suggests that two distinct regions were formed in a MT in the presence of higher concentration of PJ2.

\section{Discussion}

In the present work, we explored the role of PJ domain of MAP4 on MT dynamics using various PJ domain deletion fragments. We have shown that the partial removal of the PJ domain of MAP4 decreased polymerization and depolymerization distances, resulting in the attenuation of the dynamic instability in individual MTs. The present results are the first direct evidence for the involvement of the PJ domain of heat-stable MAPs on MT dynamics.

The truncation of PJ domain of MAP4 attenuated the dynamic instability. The attenuation is not simply due to the lengths of remaining PJ domain. The shortest, PJ2, showed the most obvious effects. However, the longest, PJ1, showed the second strongest effect and the second longest one, KDM-MTB, did not show any effect of the deletion. Moreover, different effects on MT dynamics are not simply due to the net charge in the remaining part of PJ domain. Table IV summarizes the physical properties of PJ domain in the truncated mutants. The strongest tendency was provided by $\mathrm{PJ} 2$ with the lowest charge mutants. The acidic properties are suspected to be responsible for the protruding of the PJ domain off the MT surface being charged negatively, but the order in average charge again does not correlate with the attenuation effects on MT dynamics. Rather, the present tendency in the attenuation of dynamic instability by the removal of PJ domain is similar to the tendency for the bundling formation. Although no MT bundles were formed under the present conditions, stronger effects of attenuation were obtained with constructs to induce MT bundling (Iida et al., 2002).

The present attenuation of dynamic instability appears to depend on the specific property or structure of the deleted region from PJ domain (Fig. 1). Since no obvious change in dynamic instability was observed in KDM-MTB, KDMregion is likely sufficient to confer the proper role of the PJ domain to MAP4 in the regulation of MT dynamics. The KDM-repeat region is a characteristic structure of the PJ domain of MAP4 and is a highly conserved unit among various species (Aizawa et al., 1990; Chapin et al., 1991; West et al., 1991). According to West et al. (1991), the structure of this region was predicted to be composed of a series of short $\alpha$-helices interspersed by frequent proline residues, and to form a long filamentous unit with a high degree of flexibility. This high flexibility may be sufficient to infer the effects of the PJ domain on MT dynamics in MAP4. However, complete removal of the KDM-repeat region did not lead to the strongest attenuation, which is observed in
Table IV. STRUCTURAL PARAMETERS OF PROJECTION DOMAIN OF MAP4 AND DELETION MUTANTS

\begin{tabular}{lccc}
\hline & Residue number & Net charge $^{\mathrm{a}}$ & Charge per residue $^{\mathrm{b}}$ \\
\hline wtMAP4 & 692 & -65.0 & -0.094 \\
PJ1 & 397 & -30.5 & -0.077 \\
PJ2 & 207 & -16.5 & -0.080 \\
Na-MTB & 232 & -32.5 & -0.140 \\
KDM-MTB & 375 & -26.5 & -0.071 \\
\hline
\end{tabular}

Net charge was obtained assuming that charge for aspartic acid and glutamic acid is -1 , that for histidine is +0.5 , and that for arginine and lysine is +1 . ${ }^{\mathrm{b}}$ Charge per residue was obtained by dividing net charge by residue number.

Na-MTB. It is possible that the intact Na region and/or the simultaneous removal of the b-region partly compensate(s) for the effects of the deletion of the KDM region.

In the presence of $2 \%$ glycerol, Grego et al. (2001) also observed pause phases as frequently as in our cases. They characterized the pause phases as a third category in MT dynamic instability for their precise analysis. Here, we considered that the pause phase that we observed is the extreme case of the attenuation of the dynamic instability, where length changes both in polymerization phase and depolymerization phase were too small to be detected by optical microscope. Various tubulin drugs have also been reported to attenuate the dynamic instability (Panda et al., 1995; Panda et al., 1997; Ngan et al., 2000). Their attenuation is thought to be caused by a "kinetic cap" composed of tubulin-drug complexes at MT ends, which decreases both the ON and OFF rate of the tubulin subunit. Because wtMAP4 increased polymerization velocity in individual MTs, particular MAP4 molecules localized at the MT ends must participate in the recruitment of tubulin subunits by affecting ON and/or OFF rate constants. Partial removal of the PJ domain altered the properties of MAP4, changing the kinetic parameters to cause a decrease in the polymerization velocity. These results are comparable to those obtained from the proteolytic removal of the PJ domain from MAP2 (Fellous et al., 1994). In the presence of digested MAP2, MTs are much more resistant to MT inhibitor drugs than those in the presence of tau or undigested MAP2. The PJ domain might modify the properties of the MTB domain to change the interaction with tubulin. We can also argue that the effects of the deletion of the PJ domain on the MT dynamics would be derived from the binding mode on MT surface, which is strongly affected by the protein concentration, because the attenuation of dynamic instability appeared only at higher concentrations of PJ1, PJ2 or $\mathrm{Na}$ MTB.

It may also be possible that truncations of the PJ domain alter the overall conformation of MAP4, especially at the MTB domain. Even if the present results are due to the conformational change in the entire (or partial) structure of MAP4, however, our data strongly demonstrated a novel 
role of PJ domain in MT dynamics. The PJ domain is not only the spacer between MTs in living cell (Chen et al., 1992), the scaffold of the signaling factors (Ober et al., 1989; Rubino et al.,1989; Ookata et al., 1995), or the negative regulator of the MT bundling for MAP4 (Iida et al., 2002), but also the important part of MAP4 to maintain the structure necessary for the regulation of dynamic instability. In addition, the role of the PJ domain in maintaining the entire structure of MAP4 is region-dependent, because different deletions caused different changes in dynamic instability.

Although exceptionally long depolymerization phases occurred occasionally, the attenuation effect of the deletion of the PJ domain is the strongest in PJ2, which retained the shortest PJ domain. The strongest effects might be reflected by the enhanced ability of MT-binding (Table I). Attenuation of the dynamic instability would depend on the amount of bound MAPs. Higher amounts of bound MAPs decrease in both the association rate of polymerizing tubulin and the dissociation rate of depolymerizing tubulin. Lowering the association rate would increase the probability of loss of GTP-cap resulting in the high frequency of catastrophe. Rescue events also depend on the amount of bound MAPs, because they occur at the high-density region of MAP2 along the MT lattice (Ichihara et al., 2001). However, the presence of exceptionally longer depolymerization phases is difficult to explain only by an increased amount of bound MAP. If MAP4 also has the same property as MAP2, a higher ratio of $\mathrm{PJ} 2$ to tubulin would be consistent with a decreased length of depolymerization, but inconsistent with long depolymerization.

The velocity for longer depolymerization phases was significantly faster than that for shorter ones (Table III). This means that the inhibitory effect of PJ2 on the depolymerization velocity is weaker in longer phases than in shorter phases, suggesting an uneven distribution of PJ2 along the MT length. The uneven distribution would be due to the higher cooperativity of MT binding of PJ2 than wtMAP4. Additionally, the depolymerization distance was higher in PJ2 than in MAP4 at $0.1 \mu \mathrm{M}$ (Fig. 5). This indicates that high-density regions, which are required to stop depolymerization (Ichihara et al., 2001), are more difficult to form in PJ2 than in MAP4 at lower concentrations. This supports the hypothesis that the cooperativity of binding is higher in PJ2 than in MAP4. The PJ domain of MAP4 may participate in controlling the cooperative binding. MAP2 has been reported to bind cooperatively (Wallis et al., 1993), but its MTB domain does not bind cooperatively (Coffey et al., 1995). These previous results and ours would support the idea that the PJ domain of heat stable MAP somehow participates in the cooperative binding onto the MT surface, though our results, in which the partial removal of the PJ domain from MAP4 increase the positive cooperativity, seem to be inverse to the case of MAP2. Experiments to visualize the difference in distribution of these MAP4 constructs along the MT length are now in progress in our laboratory.

Acknowledgements. This work was supported by Grants-in-Aid for Scientific Research from the Ministry of Education, Science and Culture of Japan to TJI and HH. The authors would like to thank Dr. Kenneth Ritchie of his critical reading of the manuscript. The authors are grateful to the Meat Hygiene Inspection Laboratory of Nagoya City for the help in obtaining bovine and porcine brains.

\section{References}

Aizawa, H., Kawasaki, H., Murofushi, H., Kotani, S., Suzuki, K., and Sakai, H. 1989. A common amino acid sequence in 190-kDa microtubule-associated protein and tau for the promotion of microtubule assembly. J. Biol. Chem., 264: 5885-5890.

Aizawa, H., Emori, Y., Murofushi, H., Kawasaki, H., Sakai, H., and Suzuki, K. 1990. Molecular cloning of a ubiquitously distributed microtubule-associated protein with $\mathrm{Mr}$ 190,000. J. Biol. Chem., 265: 1384913855.

Aizawa, H., Emori, Y., Mori, A., Murofushi, H., Sakai, H., and Suzuki, K. 1991. Functional analysis of domain structure of microtubule-associated protein-4 (MAP-U). J. Biol. Chem., 266: 9841-9846.

Andersen, S.S. 1999. Balanced regulation of microtubule dynamics during the cell cycle: a contemporary view. BioEssays, 21: 53-60.

Bulinski, J.C. 1994. MAP4. in Microtubules (Hyams, J.S and Lloyd, C.W., eds). Wiley-Liss, New York, pp. 167-182.

Cassimeris, L., Pryer, N.K., and Salmon, E.D. 1988. Real-time observations of microtubule dynamic instability in living cells. J. Cell. Biol., 107: $2223-2231$

Cassimeris, L. 1999. Accessory protein regulation of microtubule dynamics throughout the cell cycle. Curr. Opin. Cell. Biol., 11: 134-141.

Chapin, S.J. and Bulinski, J.C. 1991. Non-neuronal 210-103 Mr microtubule-associated protein (MAP4) contains a domain homologous to the microtubule-binding domains of neuronal MAP2 and tau. J. Cell. Sci., 98: $27-36$.

Chen, J., Kanai, Y., Cowan, N.J., and Hirokawa, N. 1992. Projection domains of MAP2 and tau determine spacings between microtubules in dendrites and axons. Nature, 360: 674-677.

Coffey, R.L. and Purich, D.L. 1995. Non-cooperative binding of the MAP-2 microtubule-binding region to microtubules. J. Biol. Chem., 270: 10351040.

Desai, A. and Mitchison, T.J. 1997. Microtubule polymerization dynamics. Annu. Rev. Cell Dev. Biol., 13: 83-117.

Dreschel, D.N., Hyman, A.A., Cobb, M.H., and Kirschner, M.W. 1992. Modulation of dynamic instability of tubulin assembly by microtubuleassociated protein tau. Mol. Biol. Cell, 3: 1141-1154.

Fellous, A., Prasad, V., Ohayon, R., Jordan, M.A., and Luduena, R.F. 1994. Removal of the projection domain of microtubule-associated protein 2 alters its interaction with tubulin. J. Prot. Chem., 13: 381-391.

Grego, S., Cantillana, V., and Salmon, E.D. 2001. Microtubule treadmilling in vitro investigated by fluorescence speckle and confocal microscopy. Biophys. J., 81: 66-78.

Gustke, N., Trinczek, B., Biernat, J., Mandelkow, E.M., and Mandelkow, E. 1994. Domains of tau protein and interaction with microtubules. Biochemistry, 33: 9511-9522.

Hashimoto, T. 2003. Dynamics and regulation of plant interphase microtubules: a comparative view. Curr. Opin. Plant Biol., 6: 568-576.

Hirokawa, N. 1994. Microtubule organization and dynamics dependent on microtubule-associated proteins. Curr. Opin. Cell Biol., 6: 74-81.12.

Horio, T. and Hotani, H. 1986. Visualization of the dynamic instability of individual microtubules by dark-field microscopy. Nature, 321: 605607. 
Ichihara, K., Kitazawa, H., Iguchi, Y., Hotani, H., and Itoh, T.J. 2001 Visualization of the stop of microtubule depolymerization that occurs at the high-density region of microtubule-associated protein 2 (MAP2). J. Mol. Biol., 312: 107-118.

Iida, J., Itoh, T.J., Hotani, H., Nishiyama, K., Murofushi, H., Bulinski, J.C., and Hisanaga, S. 2002. The projection domain of MAP4 supresses the microtubule-bundling activity of the microtubule binding domain. $J$. Mol. Biol., 320: 97-106.

Itoh, T.J. and Hotani, H. 1994. Microtubule-stabilizing activity of MAPs is due to increase in frequency of rescue in dynamic instability: shortening length decreases with binding of MAPs onto microtubules. Cell Struct. Funct., 19: 279-290.

Itoh, T.J., Hisanaga, S., Hosoi, T., Kishimoto, T., and Hotani, H. 1997. Phosphorylation states of microtubule-associated protein 2 (MAP2) determine the regulatory role of MAP2 in microtubule dynamics. Biochemistry, 36: 12574-12582.

Joly, J.C., Flynn, G., and Purich, D.L. 1989. The microtubule fragment of microtubule-associated protein 2 (MAP2): location of the proteaseaccessible site and identification of an assembly-promoting peptide. $J$. Cell Biol., 109: 2289-2294.

Kawachi, A., Ichihara, K., Hisanaga, S., Iida, J., Toyota, H., Hotani, H., and Itoh, T.J. 2003. Different protofilament-dependence of the microtubule binding between MAP2 and MAP4. Biochem. Biophys. Res. Commun., 305: 72-78.

Kindler, S., Schulz, B., Goedert, M., and Garner, C.G. 1990. Molecular structure of microtubule-associated protein $2 \mathrm{~b}$ and $2 \mathrm{c}$ from rat brain. $J$. Biol. Chem., 265: 19679-19684.

Kinoshita, K., Habermann, B., and Hyman, A.A. 2002. XMAP215: a key component of the dynamic microtubule cytoskeleton. Trends Cell Biol., 12: $267-273$.

Kowalski, R.J. and Williams, R. C. Jr. 1993. Microtubule-associated protein 2 alters the dynamic properties of microtubule assembly and disassembly. J. Biol. Chem., 268: 9847-9855.

Laemmli, U.K. 1970. Cleavage of structural proteins during the assembly of the head of bacteriophage T4. Nature, 227: 680-685.

Lewis, S.A., Wang, D., and Cowan, N.J. 1988. Microtubule-associated protein 2 (MAP2) shares a microtubule-associated binding motif with tau protein. Science, 242: 936-939.

Mandelkow, E. and Mandelkow, E.M. 1995. Microtubule and microtubule-associated proteins. Curr. Opin. Cell Biol., 7: 72-81.

Mitchison, T. and Kirschner, M. 1984. Dynamic instability of microtubule growth. Nature, 312: 237-242.

Ngan, V.K., Bellman, K., Panda, D., Hill, B.T., Jordan, M.A., and Wilson, L. 2000. Novel actions of the antitumor drugs vinflunine and vinorelbine on microtubules. Cancer Res., 60: 5045-5051.

Obar, R.A., Dingus, J., Bayley, H., and Vallee, R.B. 1989. The RII subunit of cAMP-dependent protein kinase binds to a common amino-terminal domain of microtubule-associated proteins 2A, 2B, and 2C. Neuron, 3, 639-645.
Olmsted, J.B. 1986. Microtubule-associated protein. Ann. Rev. Cell Biol., 2: $421-457$.

Ookata, K., Hisanaga, S., Bulinski, J.C., Murofushi, H., Aizawa, H., Itoh, T.J., Hotani, H., Okumura, E., Tachibana K., and Kishimoto, T. 1995. Cyclin B interaction with microtubule-associated protein 4 (MAP4) targets $\mathrm{p} 34^{\mathrm{cdc} 2}$ kinase to microtubules and is a potential regulator of M-phase microtubule dynamics. J. Cell Biol., 128: 849-862.

Panda, D., Daijo, J.E., Jordan, M.A., and Wilson, L. 1995. Kinetic stabilization of microtubule dynamics at steady state in vitro by substoichiometric concentrations of tubulin-colchicine complex. Biochemistry, 34: 9921-9929.

Panda, D., Singh, J.P., and Wilson, L. 1997. Suppression of microtubule dynamics by LY290181. J. Biol. Chem., 272: 7681-7687.

Pryer, N.K., Walker, R.A., Skeen, V.P., Bourns, B.D., Soboeiro, M.F., and Salmon, E.D. 1992. Brain microtubule-associated proteins modulate microtubule dynamic instability in vitro. Real-time observations using video microscopy. J. Cell Sci., 103: 965-976.

Rubino, H.M., Dammerman, M., Shafit-Zagardo, B., and Erlichman, J. 1989. Localization and characterization of the binding site for the regulatory subunit of type II cAMP-dependent protein kinase of MAP2. Neuron, 3: 631-638.

Sammak, P.J. and Borisy, G.G. 1988. Direct observation of microtubule dynamics in living cells. Nature, 332: 724-726.

Schulze, E. and Kirschner, M.W. 1988. New features of microtubule behavior observed in vivo. Nature, 334: 356-359.

Tokuraku, K., Katsuki, M., Nakagawa, H., and Kotani, S. 1999. A new model for microtubule-associated protein (MAP)-induced microtubule assembly in vitro: the Pro-rich region of MAP4 promotes nucleation of microtubule assembly in vitro. Eur. J. Biochem., 259: 158-166.

Trinczek, B., Biernat, J., Baumann, K., Mandelkow, E.M., and Mandelkow, E. 1995. Domains of tau protein, differential phosphorylation and dynamic instability of microtubules. Mol. Cell Biol., 6: 18871902.

Walker, R.A., O’Brien, E.T., Pryer, N.K., Soboeiro, M.F., Voter, W.A., Erickson, H.P., and Salmon, E.D. 1988. Dynamic instability of individual microtubules analyzed by video light microscopy: rate constants and transition frequencies. J. Cell. Biol., 107: 1437-1448.

Walker, R.A., Inoué, S., and Salmon, E.D. 1989. Asymmetric behavior of severed microtubule ends after ultraviolet-microbeam irradiation of individual microtubules in vitro. J. Cell Biol., 108: 931-937.

Wallis, K.T., Azhar, S., Rho, M.B., Lewis, S.A., Cowan, N.J., and Murphy, D.B. 1993. The mechanism of equilibrium binding of microtubuleassociated protein 2 to microtubules. Binding is a multi-phasic process and exhibits positive cooperativity. J. Biol. Chem., 268: 15158-15167.

West, R.R., Tenbarge, K.M., and Olmsted, J.B. 1991. A model for microtubule-associated protein 4 structure. J. Biol. Chem., 266: 21886-21896.

(Received for publication, November 22, 2004

and accepted, December 27, 2004) 